\title{
Astronomical of ${ }^{\text {Joural }}$ Telescopes, Instruments, and Systems
}

\section{In-orbit operation of the soft $x$-ray spectrometer onboard the Hitomi satellite}

\section{Masahiro Tsujimoto}

Kazuhisa Mitsuda

Richard L. Kelley

Jan-Willem den Herder

Thomas G. Bialas

Kevin R. Boyce

Meng P. Chiao

Cor P. de Vries

Michael J. DiPirro

Megan E. Eckart

Yuichiro Ezoe

Ryuichi Fujimoto

Akio Hoshino

Kumi Ishikawa

Yoshitaka Ishisaki

Caroline A. Kilbourne

Shu Koyama

Maurice A. Leutenegger

Candace M. Masters
Ikuyuki Mitsuishi

Hirofumi Noda

Takashi Okajima

Atsushi Okamoto

Frederic S. Porter

Kosuke Sato

Yohichi Sato

Joseph C. Savinell

Makoto Sawada

Hiromi Seta

Peter J. Shirron

Gary A. Sneiderman

Yoh Takei

Toru Tamagawa

Makoto S. Tashiro

Tomomi Watanabe

Shinya Yamada

Noriko Y. Yamasaki

Yoichi Yatsu 


\title{
In-orbit operation of the soft $\mathrm{x}$-ray spectrometer onboard the Hitomi satellite
}

\author{
Masahiro Tsujimoto, ${ }^{a, *}$ Kazuhisa Mitsuda, ${ }^{a}$ Richard L. Kelley, ${ }^{b}$ Jan-Willem den Herder, ${ }^{c}$ Thomas G. Bialas, ${ }^{b}$ \\ Kevin R. Boyce, ${ }^{b}$ Meng P. Chiao, ${ }^{b, d}$ Cor P. de Vries, ${ }^{c}$ Michael J. DiPirro, ${ }^{b}$ Megan E. Eckart, ${ }^{b}$ Yuichiro Ezoe, ${ }^{e}$ \\ Ryuichi Fujimoto, ${ }^{f}$ Akio Hoshino, ${ }^{9}$ Kumi Ishikawa, ${ }^{a}$ Yoshitaka Ishisaki, ${ }^{e}$ Caroline A. Kilbourne, ${ }^{b}$ Shu Koyama, ${ }^{a}$ \\ Maurice A. Leutenegger, ${ }^{\mathrm{b}, \mathrm{d}}$ Candace M. Masters, ${ }^{\mathrm{b}}$ Ikuyuki Mitsuishi, ${ }^{\mathrm{h}}$ Hirofumi Noda, ${ }^{\mathrm{i}}$ Takashi Okajima, \\ Atsushi Okamoto, ${ }^{j}$ Frederic S. Porter, ${ }^{b}$ Kosuke Sato, ${ }^{\mathrm{k}}$ Yohichi Sato,' Joseph C. Savinell, ${ }^{\mathrm{b}}$ Makoto Sawada,' \\ Hiromi Seta, ${ }^{\mathrm{e}}$ Peter J. Shirron, ${ }^{\mathrm{b}}$ Gary A. Sneiderman, ${ }^{\mathrm{b}}$ Yoh Takei, ${ }^{\mathrm{j}}$ Toru Tamagawa, ${ }^{\mathrm{m}}$ Makoto S. Tashiro, ${ }^{\mathrm{n}}$

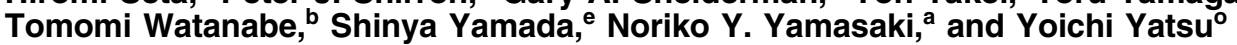 \\ ${ }^{a}$ Institute of Space and Astronautical Science, Japan Aerospace Exploration Agency, Sagamihara, Japan \\ ${ }^{b}$ Goddard Space Flight Center, National Aeronautics and Space Administration, Greenbelt, Maryland, United States \\ 'SRON Netherlands Institute for Space Research, Utrecht, The Netherlands \\ dUniversity of Maryland, Department of Astronomy, Baltimore, Maryland, United States \\ eTokyo Metropolitan University, Department of Physics, Tokyo, Japan \\ fKanazawa University, Department of Physics, Kanazawa, Japan

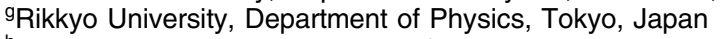 \\ ${ }^{h}$ Nagoya University, Department of Physics, Nagoya, Japan \\ 'Tohoku University, Frontier Research Institute for Interdisciplinary Sciences, Sendai, Japan \\ jJapan Aerospace Exploration Agency, Research and Development Directorate, Tsukuba, Japan \\ ${ }^{k}$ Tokyo University of Science, Department of Physics, Tokyo, Japan \\ 'Aoyama Gakuin University, Department of Physics and Mathematics, Sagamihara, Japan \\ mRIKEN Nishina Center, Wako, Saitama, Japan \\ nSaitama University, Department of Physics, Saitama, Japan \\ oTokyo Institute of Technology, Department of Physics, Tokyo, Japan
}

\begin{abstract}
We summarize all of the in-orbit operations of the soft x-ray spectrometer (SXS) onboard the ASTRO$\mathrm{H}$ (Hitomi) satellite. The satellite was launched on February 17, 2016, and the communication with the satellite ceased on March 26, 2016. The SXS was still in the commissioning phase, in which the set-ups were progressively changed. This paper is intended to serve as a concise reference of the events in orbit in order to properly interpret the SXS data taken during its short lifetime and as a test case for planning the in-orbit operation for future microcalorimeter missions. () The Authors. Published by SPIE under a Creative Commons Attribution 3.0 Unported License. Distribution or reproduction of this work in whole or in part requires full attribution of the original publication, including its DOI. [DOI: 10.1117/1. JATIS.4.1.011205]
\end{abstract}

Keywords: Hitomi; soft x-ray spectrometer; in-orbit operations; x-ray microcalorimeter.

Paper 17022SSP received May 26, 2017; accepted for publication Oct. 13, 2017; published online Nov. 6, 2017.

\section{Introduction}

The ASTRO-H observatory, ${ }^{1}$ which was renamed "Hitomi" after the successful launch on February 17, 2016 (Y + 0; hereafter, the elapsed days from the launch are labeled as $\mathrm{Y}+$ ), was decommissioned due to the loss of the attitude control and resultant breakup of the spacecraft on $\mathrm{Y}+38$. The soft $\mathrm{x}$-ray spectrometer $(\mathrm{SXS})^{2}$ onboard the spacecraft was powered on $\mathrm{Y}+0$, started $50 \mathrm{mK}$ control on $\mathrm{Y}+5$, achieved first light on $\mathrm{Y}+7$, and continued observations with superb performance ${ }^{3}$ and without any major issues until the breakup event. The commissioning of the instrument was planned to be completed in April 2016. The major remaining activities included opening of the Dewar gate valve $(\mathrm{GV})$, the use and tuning of the modulated x-ray source (MXS), ${ }^{4}$ and adjustment of the event threshold. Cryogen-free operation ${ }^{5}$ was not tested in orbit, since its use was planned for years into the mission after the liquid helium was expended. During the short lifetime, we acquired data with the SXS that have both scientific and engineering values. Many science papers have already been published ${ }^{6,7}$ or are in

*Address all correspondence to: Masahiro Tsujimoto, E-mail: tsujimot@astro .isas.jaxa.jp preparation. These data were taken with changing instrumental set-ups that were different from the planned normal configurations, which we need to consider for interpreting these data properly.

Here, we describe the SXS operations made in orbit until the end of the mission. The purpose of this paper is twofold: (1) we summarize all of the major changes made for the SXS components in orbit (Sec. 2), which serves as a concise reference for all publications based on the SXS in-orbit data. (2) We record the in-orbit operations planned in the normal phase of the SXS (Sec. 3) and summarize some lessons learned from the in-orbit operations (Sec. 4) in order to aid in planning for future microcalorimeter missions. Details are found in other articles of the same special section for the spacecraft, ${ }^{1}$ the overall mission instrument, ${ }^{2}$ the sensor and its performance,${ }^{8-11}$ the mirror and the aperture assembly, ${ }^{12,13}$ the cooling chain, ${ }^{5,14-17}$ the vibration isolation system, ${ }^{18}$ the filter wheel $(\mathrm{FW}),{ }^{4}$ and the signal processing. ${ }^{19,20}$

All data presented here are available in Ref. 21. Acronyms used in this manuscript are summarized in Table 1.

\section{Journal of Operations}

Table 2 summarizes all of the major operations in orbit, whereas Table 3 gives a summary of different set-ups for all scientific 
Table 1 Acronyms.

\begin{tabular}{|c|c|c|c|}
\hline Acronym & Descriptoin & Acronym & Description \\
\hline ADR & Adiabatic demagnetization refrigerator & ADRC & ADR controller \\
\hline AT & Autonomous commands & $\mathrm{CHD}$ & Cold head \\
\hline CMP & Compressor & DIST & power distributor \\
\hline DMS & Dewar main shell & DR & Data recorder \\
\hline FW & Filter wheel & FWE & FW electronics \\
\hline GV & Gate valve & $\mathrm{HK}$ & House keeping \\
\hline HS & Heat switch & HTR & Heater \\
\hline IVCS & Inner vapor cooled shield & JT & Joule-Thomson \\
\hline JTC & JT cooler & JTD & JTC driver \\
\hline LHP & Loop heat pipe & MAG & Magnet \\
\hline MXS & Modulated X-ray source & NTE & Night earth \\
\hline OVCS & Outer vapor cooled shield & OVI & Over current \\
\hline OVT & Over temperature & PC & Pre-cooler \\
\hline PCD & PC driver & PSP & Pulse shape processor \\
\hline RCS & Reaction control system & $\mathrm{RQ}$ & Request commands \\
\hline $\mathrm{RSH}$ & RCS safe hold & SAA & South Atlantic Anomaly \\
\hline SC & Shield cooler & SCD & SC driver \\
\hline SMU & Satellite management unit & SWR & Space wire router \\
\hline SXS & Soft X-ray spectrometer & UVC & Under voltage condition \\
\hline WSH & Wheel safe hold & $\mathrm{XBOX}$ & X-ray (amplifier) BOX \\
\hline
\end{tabular}

observations in comparison to the planned final set-up. The house-keeping (HK) telemetry plots are shown for the contact passes on $\mathrm{Y}+0$ (Fig. 1), the first two days (Fig. 2), and the remaining days (Fig. 3). From the top, the panels show (a) the telescope pointing by the right ascension, the declination, and the roll angle, (b) the total primary power of all the SXS components [SXS power distributor (SXS-DIST), SXS-SWR$\mathrm{A} / \mathrm{B}$, shield cooler driver (SCD), precooler driver (PCD), Joule-Thomson cooler driver (JTD), adiabatic demagnetization refrigerator controller (ADRC), x-ray (amplifier) BOX (XBOX), pulse shape processor (PSP)-A/B, and filter wheel electronics (FWE)], (c) the secondary power input to the mechanical cryocoolers, (d) Joule-Thomson (JT) circuit pressures or the filter wheel position, (e) magnet (MAG) current of the first- and second-stage adiabatic demagnetization refrigerators (ADRs), (f) 50-mK control temperature, (g) He tank and porous plug temperatures, (h) inner vapor-cooled shield (IVCS) temperature, and (i) the temperature difference between the saddle of the evaporator of the loop heat pipes (LHP) and the shield coolers for the four units of the SXS-LHP. These telemetry items show a wide range of activities in the complex system of the SXS. All events in Table 2 are shown with gray-dashed vertical lines, whereas the noise spectra operations (Sec. 3.2) are shown with orange-dashed vertical lines.

\subsection{Power Distributor, Cooler Drivers, SpaceWire Routers}

All of the SXS components except for the SXS-DIST were not powered at launch. The most critical operation immediately after the launch was to reestablish the low pressure of the liquid He by opening the vent valve using a stored command sequence, which required a very tight condition of the rocket being accelerated and the ambient air pressure being $<130 \mathrm{~Pa} .{ }^{15}$ The evaporated $\mathrm{He}$ is pumped out through the porous plug. In order to start it in a controlled manner, the liquid He needs some gravity while the outside environment needs to be in a low pressure. ${ }^{15}$ The resultant valve status change was confirmed in real time, as the satellite was still in the visibility range from the launch site, which was beyond our expectation.

The start of He pumping should be confirmed independently by the He tank temperature and the temperature difference between up- and down-streams of the porous plug due to the start of the vapor cooling [Fig. $1(\mathrm{~g})] .{ }^{15}$ To have these telemetry items available at the first contact, we powered on two space wire routers (SXS-SWR-A and B) and the SCD by stored commands. The He fill line was also opened. All of these were confirmed successful at the first contact $\sim 45 \mathrm{~min}$ after the launch.

The other two cooler drivers (PCD and JTD) were powered on during the second contact. The SXS-DIST, SXS-SWR-A and 
Table 2 Journal of operations and events.

\begin{tabular}{|c|c|c|c|c|c|c|c|c|c|c|c|}
\hline \multirow[b]{2}{*}{$Y^{a}$} & \multicolumn{2}{|c|}{ Date } & \multirow{2}{*}{$\begin{array}{c}\text { Time } \\
\text { (UT) }\end{array}$} & \multirow[b]{2}{*}{$C^{b}$} & \multirow[b]{2}{*}{ SXS operations } & \multirow[b]{2}{*}{$Y^{a}$} & \multicolumn{2}{|c|}{ Date } & \multirow{2}{*}{$\begin{array}{c}\text { Time } \\
\text { (UT) }\end{array}$} & \multirow[b]{2}{*}{$\mathrm{C}^{\mathrm{b}}$} & \multirow[b]{2}{*}{ SXS operations } \\
\hline & M & $\mathrm{D}$ & & & & & $M$ & $\mathrm{D}$ & & & \\
\hline \multirow[t]{19}{*}{0} & 2 & 17 & 08:45:00 & & Lift off & 4 & 2 & 21 & $08: 57: 17$ & $U$ & PSP nrec rate (2 to $1 \mathrm{~Hz} / \mathrm{CPU})$ \\
\hline & & & 08:50:20 & & He vent valve opened & & & & 09:40:48 & $S$ & ADRC calibration start \\
\hline & & & 09:02:30 & & SXS-SWR-A/B on & 5 & 2 & 22 & 03:47:59 & $U$ & $50 \mathrm{mK}$ cool down start \\
\hline & & & 09:04:00 & & SCD on & & & & 05:30 & & Cool down to $50 \mathrm{mK}$ \\
\hline & & & 09:04:40 & & He fill valve opened & & & & 05:32:55 & $U$ & First noise collection at $50 \mathrm{mK}$ \\
\hline & & & $09: 35: 13$ & $S$ & Telemetry at first contact & & & & 07:59:24 & $S$ & PSP telemetry priority change \\
\hline & & & $10: 30: 38$ & $U$ & PCD on & & & & 08:20:00 & & Detector IV curve \\
\hline & & & $10: 30: 39$ & $U$ & JTD on & & & & 15:00:00 & & Detector IV curve \\
\hline & & & $10: 30: 39$ & $U$ & JTC AT enabled & 6 & 2 & 23 & 02:09:59 & $U$ & PSP prec rate (all to $1 \mathrm{~Hz} / \mathrm{CPU}$ ) \\
\hline & & & 11:03:56 & & ADRC on & & & & 03:54:16 & $U$ & ADR recycle \#1 started \\
\hline & & & $11: 04: 16$ & & ADRC calibration start & 7 & 2 & 24 & 02:11:47 & $U$ & MNV to Perseus \\
\hline & & & 11:13:56 & $S$ & SXS-LHP heater on/off & & & & 02:20 & & First light \\
\hline & & & 11:16:39 & $S$ & SC, PC AT enabled & & & & 14:10:01 & & ADR recycle \#2 started \\
\hline & & & $11: 17: 19$ & $S$ & $\mathrm{SC}$ ramp-up to $20 \mathrm{~V} \times 2$ & 9 & 2 & 26 & 01:10:01 & & ADR recycle \#3 started \\
\hline & & & $11: 17: 19$ & $S$ & $\mathrm{PC}$ ramp-up to $18 \mathrm{~V} \times 2$ & 10 & 2 & 27 & 14:00:01 & & ADR recycle \#4 started \\
\hline & & & 12:00:38 & & ADRC calibration start & 12 & 2 & 29 & $01: 17: 41$ & S & Filter heater cold aliveness \\
\hline & & & $12: 10: 38$ & $U$ & SXS-LHP heater on/off & & & & 03:55:42 & $U$ & $\mathrm{ADRC}$ redundant $\mathrm{CAMC} \mathrm{ctrl}$ \\
\hline & & & $12: 15: 29$ & $U$ & $\mathrm{SC}$ ramp-up to $50 \mathrm{~W} \times 2$ & & & & $04: 40: 47$ & S & ADR recycle \#5 started \\
\hline & & & 13:05 & & SXS-LHP SC CHD-A stopped & 13 & 3 & 1 & $00: 32: 25$ & $U$ & IVCS, OVCS filter fingerprinting \\
\hline \multirow[t]{9}{*}{1} & 2 & 18 & $06: 14: 28$ & $S$ & $\mathrm{PC}$ ramp-up to $50 \mathrm{~W} \times 2$ & & & & 01:18:15 & $S$ & DMS filter heater control start \\
\hline & & & $07: 45$ & & SXS-LHP SC CHD-B stopped & & & & $04: 41: 27$ & S & DMS filter heater control stop \\
\hline & & & 07:53:44 & $S$ & JTC circ mode start & & & & $21: 55: 01$ & & ADR recycle \#6 started \\
\hline & & & 07:56:00 & $\mathrm{s}$ & JTC ramp-up & 14 & 3 & 2 & 04:00:00 & $U$ & PSP event thres (120 to 75 ) \\
\hline & & & $08: 51: 27$ & $S$ & JTC ramp-up & 15 & 3 & 3 & 16:00:01 & & ADR recycle \#7 started \\
\hline & & & 09:36:29 & $S$ & JTC ramp-up & 17 & 3 & 5 & 09:30:01 & & ADR recycle \#8 started \\
\hline & & & $10: 36: 18$ & $U$ & JTC ramp-up & 19 & 3 & 7 & 06:20:01 & & ADR recycle \#9 started \\
\hline & & & $11: 17: 08$ & $S$ & JTC ramp-up & 21 & 3 & 9 & 03:00:01 & & ADR recycle \#10 started \\
\hline & & & $12: 42: 19$ & & JTC off by AT & 22 & 3 & 10 & 17:00:01 & & ADR recycle \#11 started \\
\hline \multirow[t]{12}{*}{2} & 2 & 19 & 05:28:43 & $U$ & JTC circ mode start & 24 & 3 & 12 & 15:00:01 & & ADR recycle \#12 started \\
\hline & & & 05:28:51 & $U$ & JTC AT enabled except for PM & 26 & 3 & 14 & 13:30:01 & & ADR recycle \#13 started \\
\hline & & & 05:31:05 & $U$ & JTC ramp-up & & & & $19: 37: 54$ & $U$ & FWE on \\
\hline & & & $06: 13: 56$ & $S$ & XBOX-A, PSP-A on & & & & 19:45:00 & $U$ & PC-A ramp down 51 to $43 \mathrm{~W}$ \\
\hline & & & 06:14:09 & $S$ & XBOX-B, PSP-B on & 27 & 3 & 15 & 16:20:00 & $U$ & JTC ramp up 14.2 to $14.8 \mathrm{~W}$ \\
\hline & & & $06: 14: 38$ & $S$ & PSP mode change to RUN & & & & $17: 57: 28$ & $U$ & FWE small angle rotation \#1 \\
\hline & & & $07: 56: 40$ & $S$ & XBOX JFET heater on & 28 & 3 & 16 & 11:00:01 & & ADR recycle \#14 started \\
\hline & & & $07: 57: 56$ & $S$ & PSP parameter setting & 29 & 3 & 17 & $19: 41: 10$ & $U$ & FWE small angle rotation \#2 \\
\hline & & & 08:49:47 & $U$ & XBOX sensor bias on & 30 & 3 & 18 & 10:20:01 & & ADR recycle \#15 started \\
\hline & & & $09: 35: 57$ & $U$ & First noise collection & & & & $16: 18: 40$ & $U$ & FWE rotation (OPN2 to $\left.{ }^{55} \mathrm{Fe}\right)$ \\
\hline & & & 10:33:22 & $S$ & JFET AT enabled & 31 & 3 & 19 & 14:38:10 & $U$ & FWE rotation $\left({ }^{55} \mathrm{Fe}\right.$ to OPN1) \\
\hline & & & $10: 33: 46$ & 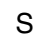 & Sensor bias $\mathrm{min} / \mathrm{max} / \mathrm{nom}$ test & 32 & 3 & 20 & $10: 20: 01$ & & ADR recycle \#16 started \\
\hline
\end{tabular}


Table 2 (Continued).

\begin{tabular}{|c|c|c|c|c|c|c|c|c|c|c|c|}
\hline \multirow[b]{2}{*}{$Y^{a}$} & \multicolumn{2}{|c|}{ Date } & \multirow{2}{*}{$\begin{array}{c}\text { Time } \\
\text { (UT) }\end{array}$} & \multirow[b]{2}{*}{$C^{b}$} & \multirow[b]{2}{*}{ SXS operations } & \multirow[b]{2}{*}{$Y^{a}$} & \multicolumn{2}{|c|}{ Date } & \multirow{2}{*}{$\frac{\text { Time }}{\text { (UT) }}$} & \multirow[b]{2}{*}{$C^{b}$} & \multirow[b]{2}{*}{ SXS operations } \\
\hline & M & $\mathrm{D}$ & & & & & M & $\mathrm{D}$ & & & \\
\hline \multirow[t]{6}{*}{4} & 2 & 21 & $03: 49: 10$ & $U$ & HS1, HS2 function test & 34 & 3 & 22 & $10: 20: 01$ & & ADR recycle \#17 started \\
\hline & & & 04:37:16 & $S$ & JTC ramp-up & 36 & 3 & 24 & 10:20:01 & & ADR recycle \#18 started \\
\hline & & & $06: 14: 52$ & $S$ & Stage 1 magnet function test & & & & $14: 45: 00$ & $U$ & JTC ramp up 14.8 to $15.2 \mathrm{~W}$ \\
\hline & & & 07:15:23 & $U$ & JTC ramp-up & & & & 16:23:07 & $u$ & FWE small angle rotation \#3 \\
\hline & & & 07:58:32 & $S$ & Stage 2 magnet function test & 37 & 3 & 25 & $13: 00: 43$ & $\mathrm{U}$ & FWE MXS HV on $(0.5 \mathrm{kV})$ \\
\hline & & & & & & 38 & 3 & 26 & $01: 41$ & & Breakup \\
\hline
\end{tabular}

aElapsed day from launch.

boperation during contact at Uchinoura (U) or Santiago (S).

Table 3 Different set-ups for SXS science observations.

\begin{tabular}{|c|c|c|c|c|c|c|c|c|c|}
\hline \multirow{2}{*}{$\begin{array}{l}\text { Seq. ID } \\
(1000-) \\
\end{array}$} & \multirow[b]{2}{*}{ Target } & \multirow{2}{*}{$\frac{\text { Start time }}{(2016, \text { UT })}$} & \multicolumn{7}{|c|}{ Conditions } \\
\hline & & & $\mathrm{T}_{\mathrm{Er}}{ }^{\mathrm{a}}$ & $\mathrm{GV}^{\mathrm{b}}$ & $\mathrm{MXS}^{\mathrm{c}}$ & Thres $^{d}$ & $P C-A^{e}$ & $\mathrm{JTC}^{\mathrm{f}}$ & $\mathrm{FW}^{\mathrm{g}}$ \\
\hline 40010 & \multirow[t]{6}{*}{ Perseus cluster } & 02-24 02:20 & $\mathrm{n}$ & c & $\mathrm{n}$ & 120 & 51 & 14.2 & OPN2 \\
\hline 40020 & & 02-25 02:14 & $\mathrm{n}$ & c & $\mathrm{n}$ & 120 & 51 & 14.2 & OPN2 \\
\hline 40030 & & 03-04 02:18 & $y$ & c & $\mathrm{n}$ & 75 & 51 & 14.2 & OPN2 \\
\hline 40040 & & 03-05 12:00 & $y$ & c & $\mathrm{n}$ & 75 & 51 & 14.2 & OPN2 \\
\hline 40050 & & 03-06 19:38 & $y$ & c & $\mathrm{n}$ & 75 & 51 & 14.2 & OPN2 \\
\hline 40060 & & 03-06 22:56 & $y$ & c & $\mathrm{n}$ & 75 & 51 & 14.2 & OPN2 \\
\hline 41010 & \multirow[t]{2}{*}{ N132D } & 03-08 02:37 & $y$ & c & $\mathrm{n}$ & 75 & 51 & 14.2 & OPN2 \\
\hline 41020 & & 03-09 01:09 & $y$ & c & $\mathrm{n}$ & 75 & 51 & 14.2 & OPN2 \\
\hline 42010 & \multirow[t]{4}{*}{ IGRJ16318-4848 } & 03-10 22:24 & $y$ & c & $\mathrm{n}$ & 75 & 51 & 14.2 & OPN2 \\
\hline 42020 & & 03-11 21:24 & $y$ & c & $\mathrm{n}$ & 75 & 51 & 14.2 & OPN2 \\
\hline 42030 & & 03-12 19:37 & $y$ & c & $\mathrm{n}$ & 75 & 51 & 14.2 & OPN2 \\
\hline 42040 & & 03-13 17:58 & $y$ & c & $\mathrm{n}$ & 75 & 51 & 14.2 & OPN2 \\
\hline 43010 & \multirow[t]{4}{*}{ RXJ1856.5-3754 } & 03-16 20:17 & $y$ & c & $\mathrm{n}$ & 75 & 43 & 14.8 & OPN2 \\
\hline 43020 & & 03-17 21:06 & $y$ & c & $\mathrm{n}$ & 75 & 43 & 14.8 & OPN2 \\
\hline 43030 & & 03-18 16:22 & $y$ & c & $\mathrm{n}$ & 75 & 43 & 14.8 & OPN2 \\
\hline 43040 & & 03-19 14:44 & $y$ & c & $\mathrm{n}$ & 75 & 43 & 14.8 & ${ }^{55} \mathrm{Fe}$ \\
\hline 50010 & \multirow[t]{4}{*}{ G21.5-0.9 } & 03-19 17:58 & $y$ & c & $\mathrm{n}$ & 75 & 43 & 14.8 & OPN1 \\
\hline 50020 & & 03-20 14:40 & $y$ & c & $\mathrm{n}$ & 75 & 43 & 14.8 & OPN1 \\
\hline 50030 & & 03-21 17:00 & $y$ & c & $\mathrm{n}$ & 75 & 43 & 14.8 & OPN1 \\
\hline 50040 & & 03-22 22:41 & $y$ & c & $\mathrm{n}$ & 75 & 43 & 14.8 & OPN1 \\
\hline 43050 & \multirow[t]{2}{*}{ RXJ1856.5-3754 } & 03-23 14:06 & $y$ & c & $\mathrm{n}$ & 75 & 43 & 14.8 & OPN1 \\
\hline 43060 & & 03-24 11:35 & $y$ & c & $\mathrm{n}$ & 75 & 43 & 14.8 & OPN1 \\
\hline 44010 & Crab & 03-25 12:36 & $y$ & c & $\mathrm{n}$ & 75 & 43 & 14.8 & OPN1 \\
\hline \multicolumn{3}{|c|}{ Planned normal set-up } & $\mathrm{y}$ & o & $y$ & TBD & 43 & 14.8 & Selectable \\
\hline
\end{tabular}

aThermal equilibrium of the instrument is reached ("y") or not (" $n$ ").

bThe gate valve is closed ("c") or opened ("o"). The open gate valve is accompanied by the temperature control of the DMS filter with a heater ${ }^{c}$ The MXS is in use ("y") or not ("n").

${ }^{\mathrm{d}}$ The event threshold. The final value was planned to be determined after the gate valve was opened.

${ }^{\mathrm{T}}$ The PC-A drive wattage in $\mathrm{W}$.

${ }^{\mathrm{f}}$ The JTC drive wattage in W.

9The position of the filter wheel. The position in the normal set-up was user selectable. 


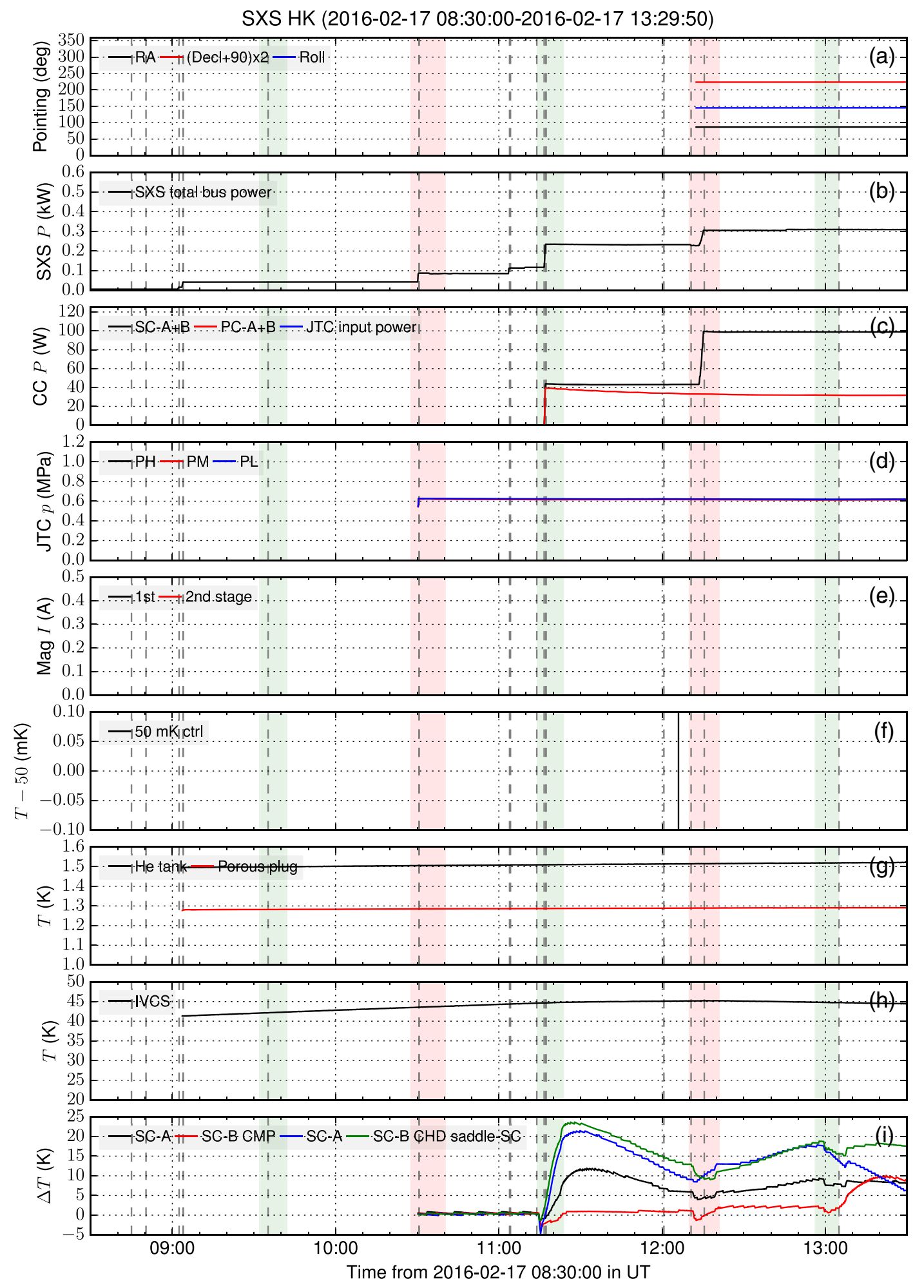

Fig. 1 (a)-(i) SXS HK plot (1) contact passes on $Y+0$. Green and magenta stripes are contacts at the Santiago or Uchinoura station.

$\mathrm{B}$, and the three cooler drivers (SCD, PCD, and JTD) were continuously operated until the breakup.

\subsection{Cryocoolers and Loop Heat Pipes}

The next urgent operation was to start the cryocooling to minimize the temperature increase of the He tank. A delay could cause a significant, or even entire, loss of the liquid $\mathrm{He}$ and thus a shortened lifetime of the mission. ${ }^{16}$ This was the most difficult part of the operation planning, as we needed to find time in limited contact passes, which were filled with many critical operations when the spacecraft was most vulnerable to accidents. On Y +0 , we started two shield coolers (SC-A and B) 
SXS HK (2016-02-17 08:00:00-2016-02-18 23:50:00)

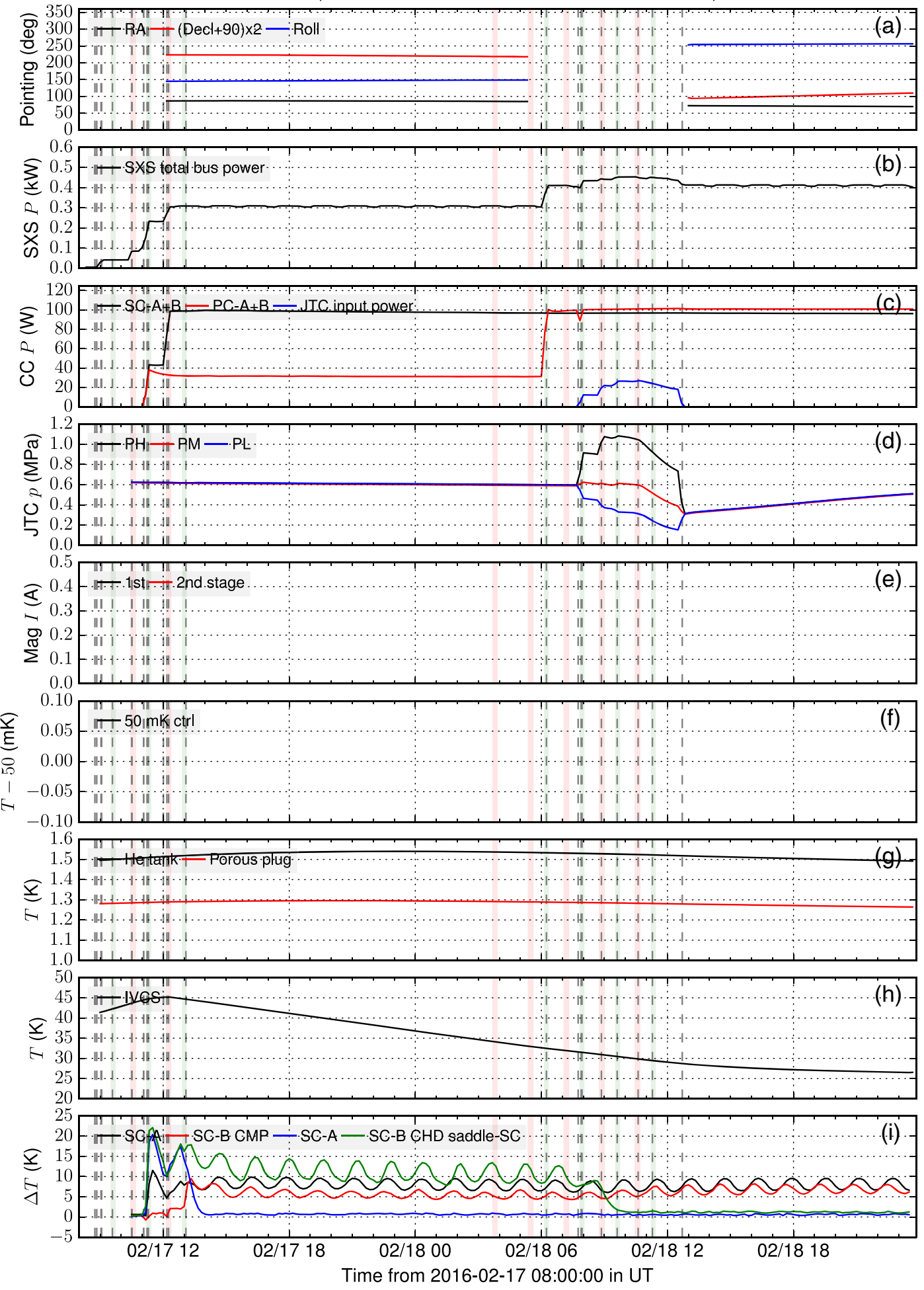

Fig. 2 (a)-(i) SXS HK plot (2) for the first two days.

with $50 \mathrm{~W}$ and two precoolers (PC-A and B) with 32-W operation [Figs. 1 and 2(c)]. This was beyond the requirement of starting just SC-A and B at $40 \mathrm{~W}$. As a result, the He tank temperature started to decrease on the first day.

On Y +1 , we started the Joule-Thomson cooler (JTC) and ramped up step by step at every contact as planned. However, by the next remote pass, JTC was turned off by an autonomous
(AT) function set for one of the JT circuit pressures [Figs. 2(c) and 2(d)]. We soon figured out that this was not a malfunction, but an expected behavior when the working $\mathrm{He}$ gas in the circuit was quickly liquefied at a certain temperature. We never experienced this during ground testing with different cooling speeds. This AT function was necessary only for the cryogen-free mode, so we restarted JTC on Y +2 after disabling this AT function. 
SXS HK (2016-02-19 00:00:00-2016-03-25 23:45:00)

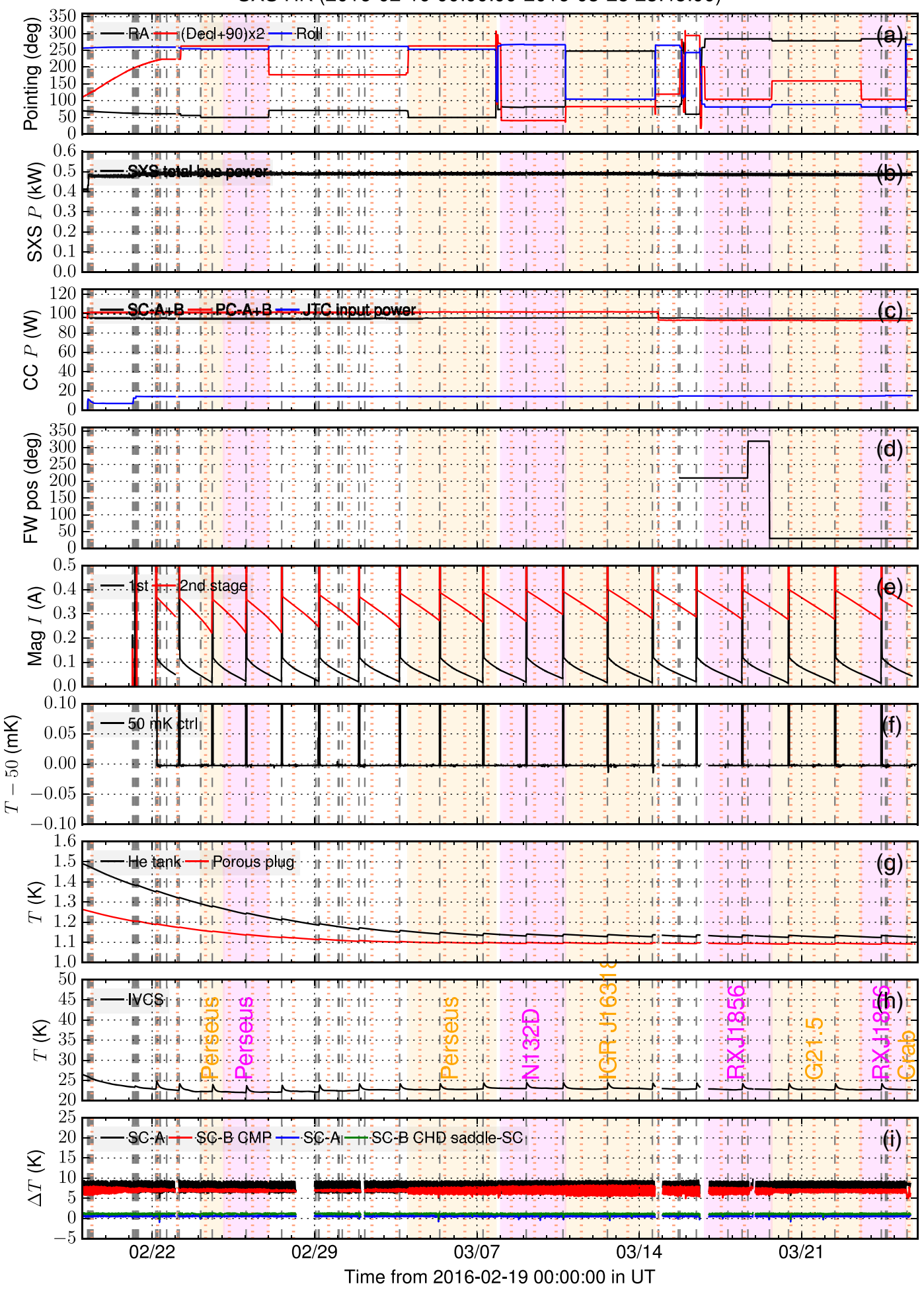

Fig. 3 (a)-(i) SXS HK plot (3) for all the remaining days until breakup. Observations of astronomical targets and noise measurements started in this phase, which are, respectively, shown with alternating colors and orange-dotted lines.

Since then, the cryocoolers showed a very stable and high performance. ${ }^{17}$ In fact, the cooling power was higher than expected presumably because the Dewar temperatures were closer to the equilibrium than ground testing. This allowed us to lower and rebalance the input powers to the coolers.
We operated JTC at $14.2 \mathrm{~W}$, which was much lower than the nominal power $(25.2 \mathrm{~W})$ on the ground until $\mathrm{Y}+26 / 27$. By utilizing this extra margin, we increased the power to $14.8 \mathrm{~W}$ for JTC and decreased it from 51 to $43 \mathrm{~W}$ for PC-A [Figs. 3(c)]. This was because the compressor temperature of 
PC-A was higher than the others, and the decreased wattage would help to prolong the lifetime with a lower out-gassing rate at a lower temperature.

The SXS had four sets of LHP (SXS-LHP) to support heat dissipation from the shield-cooler compressors (SC-A/B CMP) and their cold heads (SC-A/B CHD). All four units were started successfully with a use of preconditioning and start-up heaters before the ramp-up of $\mathrm{SC}$ on $\mathrm{Y}+0$. The two units for CMP worked until the end, but the other two finished working 1.0 and $19.5 \mathrm{~h}$ later for SC-A CHD and SC-B CHD, respectively [Figs. 2(i)]. This was expected as the heat input was too small for the LHP to keep working. The LHP was not necessary with a low-heat input at the beginning of the mission, and we left the units as they were.

\subsection{Adiabatic Demagnetization Refrigerator Controller}

The ADRC was powered on $\mathrm{Y}+0$ and the thermometers were calibrated for precise temperature monitor and control of the low-temperature stages. The function test was performed for the heat switches (HS) and the ADR magnet current control on $\mathrm{Y}+4$, and the $50-\mathrm{mK}$ control was started on $\mathrm{Y}+5$. Since then, a total of 18 ADR recycles were performed successfully, ${ }^{14}$ which are visible in Figs. 3(e)-3(h). The function and the control of the third-stage ADR for the cryogen-free operation were not tested in orbit.

The ADRC also controls the heaters on the optical blocking filters. ${ }^{8}$ The heaters for both the IVCS and outer vapor-cooled shields (OVCS) filters were checked for their aliveness on $\mathrm{Y}+13$. The temperature control of the Dewar main shell (DMS) filter heater was tested for $3.5 \mathrm{~h}$ on $\mathrm{Y}+13$. The DMS filter heater control was planned to be restarted permanently a few days before the GV open to protect the filter from contamination.

\subsection{Signal Processing Electronics}

On $\mathrm{Y}+2$, we powered on both the analog and digital signal processing electronics (XBOX ${ }^{2,9}$ and PSP, ${ }^{19}$ respectively). We started the temperature control of the JFET and the bias input to the sensors with XBOX. The CPU program of the PSP was started and the initial parameters were set for the signal processing. Noise measurement (Sec. 3.2) and bias test were performed on the same day with the detector at $\sim 1 \mathrm{~K}$, confirming no problem. On $\mathrm{Y}+5$, we measured the noise of the detector at $50 \mathrm{mK}$ for the first time in orbit. The detector $I-V$ curve was also measured. No change was found in comparison to the ground results. ${ }^{3,13}$

Some PSP parameters needed adjustment in orbit because we did not know the precise noise level and the consumption rate of the data recorder (DR) during the South Atlantic Anomaly (SAA) passages. After the successful first light observation, we lowered the event threshold from 120 to 75 (equivalent to 166 to $104 \mathrm{eV}$ ) on $\mathrm{Y}+14$ as the noise level was low enough. The final threshold was planned to be fixed after the GV was open.

On $\mathrm{Y}+4$ to 6 , we adjusted the rate of pulse and noise record dumps. The default settings were too high, which resulted in the loss of noise record data between around February 19 19:50 to February 21 3:45 with a full usage of the DR. We also changed the priorities of data when writing into the DR (data with a lower priority are overwritten by those with a higher priority) so that we could record the diagnostic and event data at a higher priority than the others.

\subsection{Filter Wheel}

The FWE was the last SXS component to be powered on $\mathrm{Y}+26$. FWE controls the FW and the MXS. For the commissioning of the $\mathrm{FW}$, we executed a rotation by a small angle ( $\pm 2 \mathrm{deg}$ ) for the first time on $\mathrm{Y}+27$ and started the weekly maintenance operation on $\mathrm{Y}+29$ and 36 (Sec. 3.2). A large angle (60 deg or $120 \mathrm{deg}$ ) rotation to change the filters was not originally planned until it was required during the performance verification phase. However, in order to calibrate the absolute gain of individual pixels before opening the GV and using the MXS, ${ }^{10,11}$ we changed the position to illuminate the detector array with $\mathrm{x}$-rays using the ${ }^{55} \mathrm{Fe}$ sources on the wheel for $22.3 \mathrm{~h}$ on $\mathrm{Y}+30$ and 31 [Fig. 3(d)]. This data set became the only reference for the absolute gain of the array pixels in orbit.

On Y +37 , we started a two-week campaign of commissioning the MXS. The high voltage of $11.3 \mathrm{kV}$ was much higher than other high voltages employed in the spacecraft, and we carefully designed a plan to ramp-up with a step-by-step verification. The first day was successful with the lowest voltage $0.5 \mathrm{kV}$ ramp-up. The second day was not started due to the communication loss by the breakup.

\section{In-Orbit Operation Plan}

The operation of the SXS was planned to be as simple as possible with the assumption that any misoperation could cause damage and that any malfunctions may not be responded to until real-time contact is available as dictated by the ground station schedule. Thus, (i) we implemented automated responses (Sec. 3.1) necessary to protect flight hardware by themselves, (ii) we designed the hardware, so that they continue observations safely without ground commands in various orbital environmental conditions, and (iii) we minimized user-selectable observational parameters (only the selection of filters was open to choose). As a result, the list of routine operations (Sec. 3.2) is short considering the complexity of the instrument.

\subsection{Automated Responses}

Many automated responses for safety are designed and implemented within a component, and the details can be found in papers describing the individual SXS components. Some components assume that others are working properly, especially those responsible for the multistage cooling chain, thus system-wide protections were needed in addition to componentlevel protections. We thus implemented and tested such automated responses using the autonomous (AT) and request (RQ) functions of the satellite management unit (SMU), or the data handling system.

Using the AT functions, SMU can send a command sequence based on the telemetry it collects from all onboard components. Using the RQ functions, any flight hardware can request the SMU to send a command sequence to itself and the others based on its own judgment. We used AT to protect the SXS hardware based on their own telemetry and RQ to protect the SXS hardware using the spacecraft information (low battery condition and loss of spacecraft attitude control) at a sampling rate of $1 \mathrm{~Hz}$. Table 4 tabulates the AT and RQ functions that were actually implemented. The JFET voltage AT is intended 
to protect the hardware from He leakage in the guard vacuum, which can be recognized as an excessively high voltage of the heaters that control the JFET temperature at $130 \mathrm{~K}$. The wheel safe hold (WSH) and reaction control system (RCS) safe hold (RSH) RQ is intended to protect from the loss of the Sun, which could come in the line of sight to bring excessive heat input to the system.

\subsection{Routine Operation}

As the mission ended before we completed the commissioning of the SXS, only a part of the routine operations was started. Here, we describe the planned routine operations for reference.

\subsubsection{Filter wheel rotations}

For the SXS, the only user selectable parameter was the choice of a filter among an open position, an open position with a ${ }^{55} \mathrm{Fe}$ source, a neutral density filter with $\sim 25 \%$ transmission, and a Be filter. It was planned to execute these rotations in the performance verification phase when observing very bright sources.

After the turn-on of the FWE, the FW was planned to be rotated \pm 2 deg once every week during a ground contact to verify its proper functioning. By the time of the spacecraft breakup, this was performed three times.

\subsubsection{Adiabatic demagnetization refrigerator recycling}

ADR should be recycled before the 50-mK hold time expires. The hold time is a function of the He tank temperature, ${ }^{14}$ which kept decreasing to an equilibrium ${ }^{16,17}$ in the first few weeks. The hold time became longer and longer at every recycle.

$\mathrm{ADRC}$ has a function to start ADR recycling automatically when the first-stage magnet current is close to $0 \mathrm{~A}$. This function was not enabled in its entire lifetime. The $50-\mathrm{mK}$ control is lost during the recycling for $\sim 1 \mathrm{~h}$. In the initial operation, we executed all the ADR recycles by command at a specified time to minimize the impact to observations. Also, in the normal phase, we planned to decide the recycling times based on the observing efficiency.

\subsubsection{Noise collections}

A set of noise data was obtained by PSP once per day during night Earth (NTE) occlusions. When NTE was not available, the data were taken in other conditions. The times of SAA passages, ADR recycles, and the spacecraft maneuvers were avoided. The noise data set consists of the following, which usually took a few minutes for collection. The noise was collected 34 times in the orbit (Fig. 3).

Table 4 Automated responses.

\begin{tabular}{|c|c|c|c|}
\hline Type & Label & Condition & Response \\
\hline \multirow[t]{14}{*}{ AT } & SC-A OVI & Over-current of SC-A & Turn off SC-A \\
\hline & SC-B OVI & Over-current of SC-B & Turn off SC-B \\
\hline & PC-A OVI & Over-current of PC-A & Turn off PC-A and JTC \\
\hline & PC-B OVI & Over-current of PC-B & Turn off PC-B and JTC \\
\hline & JTC OVI & Over-current of JTC & Turn off JTC \\
\hline & SC-A OVT & SC-A compressor temperature $>313 \mathrm{~K}$ & Turn off SC-A \\
\hline & SC-B OVT & SC-B compressor temperature $>311 \mathrm{~K}$ & Turn off SC-B \\
\hline & PC-A OVT & PC-A compressor temperature $>313 \mathrm{~K}$ & Turn off PC-A and JTC \\
\hline & PC-B OVT & PC-B compressor temperature $>313 \mathrm{~K}$ & Turn off PC-B and JTC \\
\hline & JTD I & JTD CMP-L input current $>3.3 \mathrm{~A}$ & Turn off JTC \\
\hline & JTC PL & JT circuit low pressure $<0.103 \mathrm{MPa}$ & Turn off JTC \\
\hline & JTC PM ${ }^{a}$ & JT circuit middle pressure $<0.380 \mathrm{MPa}$ & Turn off JTC \\
\hline & DMS heater ${ }^{b}$ & DMS filter heater current $>70 \mathrm{~mA}$ & Turn off the heater \\
\hline & JFET V & JFET heater voltage $>10 \mathrm{~V}$ & Turn off $X B O X$ and $A D R C$ output \\
\hline \multirow[t]{3}{*}{$\mathrm{RQ}$} & UVC & Under voltage condition & $\begin{array}{l}\text { Turn off ADRC, XBOX, PSP, FWE, } \\
\text { and JTC. Ramp down PC }\end{array}$ \\
\hline & WSH & Wheel safe hold & DMS filter heater and MAG current off \\
\hline & $\mathrm{RSH}$ & RCS safe hold & DMS filter heater and MAG current off \\
\hline
\end{tabular}

aThis was not enabled after the JTC turn-off on $Y+1$ (Sec. 2.2).

${ }^{\mathrm{b}}$ This was enabled only during the DMS filter heater control on $Y+13$ (Sec. 2.3). 


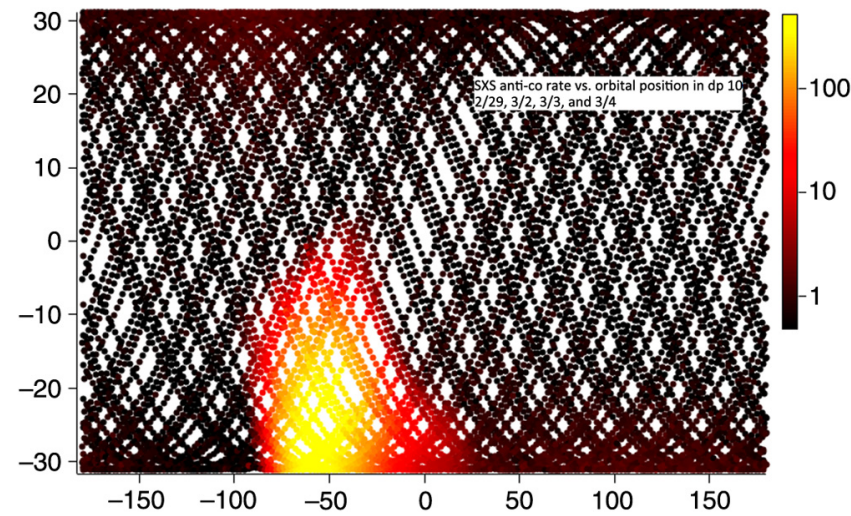

Fig. 4 Definition of SAA based on the anti-co count rate of the SXS.

- Short (1024 sample length) noise spectra from 100 noise records for all 36 pixels. Here, a sample length is $80 \mu \mathrm{s}$. A noise record is continuous samples without events.

- Long $(8 \times 1024$ sample length $)$ noise spectra from 50 noise records for all pixels except for pixels 11, 12, and 13. For the three pixels, 10 records were used due to the constantly high count rate by the ${ }^{55} \mathrm{Fe}$ source on the calibration pixel (pixel 12) and its cross-talk on the neighboring pixels (11 and 13).

- 50 sample dumps for all 36 pixels and 2 anti-co channels at the same time. Here, one sample dump is a dump of continuous data of 1024 samples regardless of whether it includes events or not.

- Twice of $5 \mathrm{~s}$ continuous data for all 36 pixels (p0 to p35) and 2 anti-co (ac-a and ac-b) channels. Two channels per CPU are dumped at the same time.

\subsubsection{Parameter and memory dumps}

Some onboard memory contents were not included in the regular $\mathrm{HK}$ telemetry. We performed weekly dumps from such memory areas from ADRC and FWE. Also, we dumped the parameters, templates, and average pulses from PSP for each observation so that users can track the set-up used for onboard pulse processing.

\subsubsection{South Atlantic Anomaly passages}

During the SAA, the temperature reading becomes noisy and the rate of false events increases. However, all the SXS hardware were designed to require no operations to cope with these changes, which worked as expected. For additional safety, we decided to ramp down the high voltage of MXS during SAA passages. The MXS commissioning was started only one day before the breakup, and the operation of ramping down at SAA ingress and up at SAA egress was tested only very partially.

The extent of the SAA used for operations is defined based on the anti-co count rate of $>3 \mathrm{~s}^{-1}$ above $30 \mathrm{keV}$ for the SXS in the pipeline processing. The map (Fig. 4) is slightly different from the one conventionally used for Suzaku.

\section{Lessons Learned}

After the mission ended, the team summarized a long list of lessons learned. The majority of the lessons learned from the in-orbit operation of Hitomi were at the mission level or related to programmatic limitations, which are beyond the scope of this paper. Overall, the in-orbit operation of the SXS instrument was quite successful. Some of the SXS lessons learned from the in-orbit operations are related to specific subsystems, and these are addressed in accompanying instrument papers, which include calibration ${ }^{10}$ and CSI performance ${ }^{3}$ and in-orbit calibration papers to be published in a special edition of the publications of the Astronomical Society of Japan. There were a few minor SXS operation issues that were unexpected and have been addressed in Sec. 2; we review these below, in addition to other SXS operations lessons learned.

- Unexpected automated power down of the JTC (Sec. 2.2). While this behavior was easily explained and the automated shut down was in fact a behavior consistent with the AT command logic, this can serve as a general caution that since the thermal environment will be different in orbit, all AT commands should be verified by simulation for a variety of operating conditions, beyond those encountered during ground testing.

- Loss of noise records for $\sim 40 \mathrm{~h}$ on the third day of flight when the detector was still warm (Sec. 2.4). There were only monitoring contacts (no commanding and data downlink) on February 19 to give a rest for the operation team, which delayed our identifying this issue. This loss of data could have been prevented by measuring the actual data rate and implementing a more appropriate setting before we started a long monitor-only period. This was not considered a high priority when the detector was warm but should have been anticipated and corrected prior to launch. In general, the prioritization scheme of the DR for SXS HK, science data, and diagnostic data should have been better-simulated and reviewed prior to launch.

- Conduct more extensive and detailed rehearsals of the initial flight operations, including unexpected behavior or data, prior to launch. These rehearsals should include the instrument teams and would have been useful for identifying tools and communication protocols needed for flight.

\section{Summary}

The SXS was started and operated successfully with no major issues in orbit, becoming the first X-ray microcalorimeter in orbit to make x-ray observations of celestial sources. Data obtained in the short mission lifetime are precious for future missions and are available to the public. These data sets were taken during the commissioning phase under various operating conditions. We described all of the SXS commissioning activities and operating conditions (Tables 2 and 3), so that the data are interpreted properly. We also discussed the planned normal operation of the SXS in orbit and technical lessons learned in the orbit operation as a reference for future missions.

\section{Disclosures}

The authors have no relevant financial interests in the manuscript and no other potential conflicts of interest to disclose. 


\section{Acknowledgments}

This work was supported by JSPS KAKENHI under Grant Nos. 15H03642, 16K05309, and 24105007.

\section{References}

1. T. Takahashi et al., "The ASTRO-H (Hitomi) x-ray astronomy satellite," Proc. SPIE 9905, $99050 \mathrm{U}$ (2016).

2. R. L. Kelley et al., "The ASTRO-H high resolution soft x-ray spectrometer," Proc. SPIE 9905, 99050V (2016).

3. F. S. Porter et al., "In-flight performance of the soft x-ray spectrometer detector system on ASTRO-H," Proc. SPIE 9905, 99050W (2016).

4. C. P. de Vries et al., "Calibration sources and filters of the soft x-ray spectrometer instrument on the Hitomi spacecraft," J. Astron. Telesc. Instrum. Syst. 4, 011204 (2017).

5. G. A. Sneiderman et al., "Cryogen-free operation of the soft x-ray spectrometer instrument," Proc. SPIE 9905, 99053N (2016).

6. Hitomi Collaboration et al., "The quiescent intracluster medium in the core of the Perseus cluster," Nature 535, 117-121 (2016).

7. F. A. Aharonian et al., "Hitomi constraints on the $3.5 \mathrm{keV}$ line in the Perseus galaxy cluster," Astrophys. J. Lett. 837, L15 (2017).

8. C. A. Kilbourne et al., "The design, implementation, and performance of the ASTRO-H SXS calorimeter array and anti-coincidence detector," Proc. SPIE 9905, 99053L (2016).

9. M. P. Chiao et al., "System design and implementation of the detector assembly for the ASTRO-H soft x-ray spectrometer," Proc. SPIE 9905, 99053M (2016).

10. M. A. Leutenegger et al., "In-flight verification of the calibration and performance of the ASTRO-H (Hitomi) soft x-ray spectrometer," Proc. SPIE 9905, $99053 \mathrm{U}$ (2016).

11. M. E. Eckart et al., "Ground calibration of the ASTRO-H (Hitomi) soft x-ray spectrometer," Proc. SPIE 9905, 99053W (2016).
12. T. Okajima et al., "First peek of ASTRO-H soft x-ray telescope (SXT) in-orbit performance," Proc. SPIE 9905, $99050 \mathrm{Z}$ (2016).

13. C. A. Kilbourne et al., "The design, implementation, and performance of the ASTRO-H SXS aperture assembly and blocking filters," Proc. SPIE 9905, 99053Q (2016).

14. P. J. Shirron et al., "Design and on-orbit operation of the adiabatic demagnetization refrigerator on the Hitomi soft x-ray spectrometer instrument," Proc. SPIE 9905, 990530 (2016).

15. Y. Ezoe et al., "Porous plug phase separator and superfluid film flow suppression system for the soft x-ray spectrometer onboard ASTROH,” Proc. SPIE 9905, 99053P (2016).

16. H. Noda et al., "Thermal analyses for initial operations of the soft x-ray spectrometer (SXS) onboard ASTRO-H," Proc. SPIE 9905, 99053R (2016).

17. R. Fujimoto et al., "Performance of the helium Dewar and cryocoolers of ASTRO-H SXS," Proc. SPIE 9905, 99053S (2016).

18. Y. Takei et al., "Vibration isolation system for cryocoolers of soft x-ray spectrometer (SXS) onboard ASTRO-H (Hitomi)," Proc. SPIE 9905, 99050X (2016).

19. Y. Ishisaki et al., "In-flight performance of pulse processing system of the ASTRO-H soft x-ray spectrometer," Proc. SPIE 9905, 99053T (2016).

20. L. Angelini et al., "ASTRO-H data analysis, processing and archive," Proc. SPIE 9905, 990514 (2016).

21. https://heasarc.gsfc.nasa.gov/docs/hitomi/archive/ for detail.

Masahiro Tsujimoto received his BS, MS, and PhD degrees in physics and astrophysics from Kyoto University in 1998, 2000, and 2003, respectively. $\mathrm{He}$ is a research associate at Japan Aerospace Exploration Agency, Institute of Space and Astronautical Science. His current research interests include high-energy astrophysics and its instrumentation.

Biographies for the other authors are not available. 